\title{
MedienPädagogik
}

Zeitschrift für Theorie und Praxis der Medienbildung

\section{Überlegungen zum Lernen mit und über Medien im Zeitalter der Digitalisierung}

Heinz Moser

\section{Zusammenfassung}

Auch wenn die Medienpädagogik als erziehungswissenschaftliche Disziplin erst seit den 70er Jahren des 20. Jahrhunderts existiert, sind Fragen nach dem Lernen mit und über Medien schon weit früher diskutiert worden. Schule als Institution des Lernens war immer schon mit Medien verbunden, welche die direkte Anschaulichkeit der Welt über Fibeln und Lesebücher mit den darauf bezogenen kognitiven Strukturen verbanden und so «Lernen» ermöglichten. Lesen und Vorlesen beruhen auf Medien, mit denen Erfahrungen mit der Welt versprachlicht und damit gleichsam auf der Basis von Buchstaben «abstrakt» gemacht werden können. Der Abacus als didaktisches Hilfsmittel ist z. B. ein Medium, um den Begriff der Zahlen anschaulich zu machen und das Lernen von Zahlen zu unterstützen. Die Entwicklung der Medienpädagogik als eigene Fachdisziplin ist dann aber erst mit den technischen Medien aufgekommen - dies zuerst auf einer analogen Basis wie Film, Radio, Fernseher, Kinderkassetten etc. in diesem Beitrag soll aufgezeigt werden, wie sich die Situation der Medienpädagogik im Rahmen der Digitalisierung nochmals verändert hat und welche neuen Konzepte und Perspektiven damit verbunden sind. Dabei soll deutlich werden, wie manche der alten Überlegungen wie z. B. das Programmieren in neuem Gewand wieder erscheinen, und wie sich Medienpädagogik im Zeitalter der Digitalisierung verändern muss, wenn sie eine Zukunft haben will. 


\title{
Reflections on learning with and about media in the age of digitalisation
}

\begin{abstract}
Even though Media Education as an educational discipline has only existed since the 1970s, questions about learning with and about media have been discussed much earlier. School as an institution of learning has always been connected with media, which linked the direct vividness of the world via text and reading books with the cognitive structures related to it and thus made "learning" possible. Reading and reading aloud are based on media with which experiences with the world can be verbalised and thus made "abstract". The Abacus as a didactic tool is, for example, a medium to make the concept of numbers vivid and to support the learning of numbers. The development of Media Education as a discipline, however, only emerged with technical media - initially on an analogue basis such as film, radio, television, children's cassettes, etc. This paper will show how the situation of Media Education has changed again in the context of digitalisation and what new concepts and perspectives are associated with it. In doing so, it will become clear how some of the old considerations, such as programming, reappear in a new guise, and how Media Education must change in the age of digitalisation if it wants to have a future.
\end{abstract}

\section{Vorbemerkung: Der Beginn der Medienpädagogik als Fachdisziplin}

Die Entwicklung der Medienpädagogik als eigene Fachdisziplin ist erst mit den technischen Medien aufgekommen - dies zuerst auf einer analogen Basis wie Film, Radio, Fernseher, Hörspielkassetten etc. Diese damals «neuen» technischen Medien wurden mit Rousseau häufig als unerwünschte Miterzieher im «natürlichen Alltag» betrachtet, die von aussen kamen, in die erzieherischen Milieus der Familie eindrangen und Fragen zur Folge hatten, wie:

- Erhalten Kinder mit den Medien Zugang zu Erfahrungen, die für ihre Entwicklung noch verfrüht sind? 
- Wie kann man die Fernseh- und Medienzeit der Kinder angesichts der Informationsflut begrenzen?

- Wie kann man Kinder weg von den Schundprodukten zum «guten» Buch oder Film führen?

- Unter welchen Umständen sind Medienverbote angebracht?

- Soll man Kindern Geschichten selbst erzählen, weil sie ihre erzieherische Wirksamkeit verlieren, wenn sie von technischen Mitteln wie Kassette oder Video ersetzt werden?

Es ging also auf der einen Seite um die Inhalte, die unter dem Stichwort des «Schund» moralische Massstäbe herausforderten, dann aber auch um die erzieherische Kontrolle, die man infolge der technischen Medien zu verlieren drohte. Eine frühe bewahrpädagogische Medienpädagogik suchte denn auch Regeln, nach denen sich Kinder und Jugendliche «gefahrlos» mit der externen Sphäre der Medien verbinden durften, ohne dass sie davon negativ beeinflusst wurden. So kritisiert Ulrich Beer, Protagonist einer konservativen Medienpädagogik in den 6oer Jahren des letzten Jahrhunderts:

«Kaum ein Elternpaar bleibt gegenüber dem Betteln des Kindes auf die Dauer hart. Müdigkeit in der Schule ist die Folge, so dass der Lehrer schon auf den ersten Blick die Kinder, die zu Haus ferngesehen haben, an ihren schwimmenden Augen erkennt, die den Bildschirm noch am nächsten Tag zu spiegeln scheinen» (Beer 1960, 42).

Als Gegenmittel empfahl man Eltern noch bis ins 21. Jahrhundert hinein, gemeinsam mit den Kindern Fernsehprogramme auszuwählen, den Medienkonsum zeitlich zu beschränken, gewalttätige Medienangebote mit Verboten zu belegen. Kampagnen für das «gute» Buch und für die qualitativ als hochwertig angesehenen Fernsehangebote der öffentlich-rechtlichen Fernsehsender sollten für «kindgerechte» Inhalte sorgen.

Mit der zunehmenden Digitalisierung am Ende des letzten Jahrhunderts verschob sich die Kritik und Skepsis gegenüber den Medien nicht grundlegend. Die Mediensucht, von der z. B. Manfred Spitzer (2012) schreibt, ist nicht weit von den Überlegungen Beers entfernt. Viele Vertreterinnen und Vertreter der Medienpädagogik passten die Regeln der «analogen 
Zeit» erst einmal auf die sog. «neuen Medien» an - auf PC, Spielkonsolen, den Gebrauch des Handys etc. Denn die Medien als «geheime Miterzieher» (Beer 1960) wurden noch verstärkt zu Konkurrenten der häuslichen Erziehungsvorstellungen, je intensiver die digitalisierten Medien Teil eines medial verfassten Lebens wurden, in das wir alle eingebunden sind. Angesichts der Mediatisierung erschien es allerdings zunehmend schwieriger solche bewahrpädagogischen Regeln noch durchzusetzen.

Diese an der Position der besorgten Erziehenden orientierte Wirksamkeitsvorstellung, welche die Nutzung der Medienangebote durch Kinder regulieren will, geht davon aus, dass die Medien der Kommunikation von einem Sender zu einem oder mehreren Empfängern dienen. Petko weist in seiner «Mediendidaktik» jedoch darauf hin, dass Medientheorien, die sich vor allem als Übermittlungstheorien verstehen, im Kontext digitaler $\mathrm{Me}$ dien zu eng gedacht sind (Petko 2014, 14 ff.). Die Medien sind nicht mehr wie im analogen Zeitalter auf eine externe Sphäre des Alltags beschränkt, bei der ein klar definierter Sender aufgesucht werden muss (z. B. durch den Kauf einer Zeitung, den Besuch eines Kinos, den Fernseher im eigenen Wohnzimmer, das Festnetztelefon im Flur etc.), sondern sie sind omnipräsent und in diesen Alltag quasi eingebaut.

\begin{tabular}{|l|l|}
\hline \multicolumn{2}{|l|}{ Die Integration der Medien im Alltag } \\
\hline $\begin{array}{l}\text { Analoge Medien } \\
\text { Externe Medien beeinflussen von } \\
\text { aussen }\end{array}$ & $\begin{array}{l}\text { Digitale Medien } \\
\text { Unser Alltag ist Teil eines medial } \\
\text { verfassten Lebens }\end{array}$ \\
\hline Medien kommen von aussen & $\begin{array}{l}\text { Wir sind im Alltag in eine digitalisierte } \\
\text { Welt eingebunden }\end{array}$ \\
\hline $\begin{array}{l}\text { Medien nehmen einen definierten Teil } \\
\text { des Alltags ein }\end{array}$ & Medien sind omnipräsent \\
\hline $\begin{array}{l}\text { Medien stellen die Kommunikation mit } \\
\text { der durch sie interpretierten Aussen- } \\
\text { welt her (Radio, Fernsehen, Video etc.) }\end{array}$ & $\begin{array}{l}\text { Medien sind im Alltag «eingebaut»: «In- } \\
\text { ternet der Dinge», Smartphone etc.) }\end{array}$ \\
\hline
\end{tabular}

Tab. 1.: Die Integration von analogen und digitalen Medien im Alltag (Moser 2019, 205). 
Das Smartphone ist inzwischen zu unserem allgegenwärtigen Begleiter geworden und das Internet der Dinge nimmt auf die Möglichkeiten unseres Handelns quasi hinter unserem Rücken Einfluss. Wir gehen zwar davon aus, dass wir Informationen recherchieren, wenn wir etwas wissen wollen. Doch wie unsere Suche im Netz präpariert wird, und welches Angebot wir dann auf den Bildschirm kriegen, das ist von den Algorithmen der Suchmaschinen bereits vorbestimmt. Und dennoch: Je mehr das Internet in die alltäglichen Tätigkeiten eingebunden ist, desto weniger können wir auf entsprechende Medien wie das Smartphone verzichten - etwa, wenn Sicherheitsabfragen bei Geldgeschäften zusätzlich zwingend einen Sicherheitscode, der mit dem Smartphone zu erzeugen ist, verlangen. Es könnte in Zukunft ein Problem werden, wie weit Personen noch «geschäftsfähig» bleiben, wenn sie nicht mit den «neuen» Medien umgehen können oder wollen.

\section{Lernen mit Medien in der Schule}

Nach diesen allgemeinen Vorbemerkungen wende ich mich der Schule und der Frage des Lernens mit digitalen Medien zu: Wie bereits dargestellt, ist Lernen in der Schule immer an Medien geknüpft gewesen. Im Kontext dieses 17. Jahrbuch Medienpädagogik (Rummler u. a. 2020) sind es allerdings die Fragen nach der Veränderung des Lernens durch «technische» Medien, die im Mittelpunkt stehen. Im analogen Zeitalter waren dies u. a. Medien wie Lernfilme, Diabilder, Projektionen auf dem Epidiaskop, später dann Schulfunk, Fernsehen und Videofilme. Ab den 1980er Jahren erfolgte dann zunehmend ein Übergang in Richtung digitaler Medien.

In der Schule wurden technische Medien indessen meist nur sporadisch eingesetzt - etwa indem ein Spielfilm vor den Ferien gezeigt wurde oder indem eine Schulfunksendung vom Tonband abgespielt wurde. Didaktisch ging es dabei nicht um eine zentrale Vermittlung von Lernstoff; vielmehr wollte man Schülerinnen und Schülern damit immer wieder einmal ein - oft visuelles - ausserordentliches Erlebnis ermöglichen. Medienpädagogisch steckte dahinter manchmal auch der erhobene Zeigefinger, welcher den qualitativ positiv gewerteten Schulfunkfilm dem eher negativ konnotierten Unterhaltungsfilm aus der Alltagswelt entgegensetzte. 


\subsection{Die erste Welle offensiver technischer Medien für die Schule}

Ein häufiger und breiter Einsatz technischer Medien war ab den 1960er Jahren mit dem Overhead-Projektor verbunden, der auf analoger Basis die Wandtafel ergänzte bzw. sogar ersetzte. In einer Werbepublikation heisst es dazu in der Zeitschrift «schweizer schule»:

«Der Hellraumprojektor stellt gleich eine ganze Reihe von visuellem Material - einfach und komplex - bereit, um die Lücke zwischen den hochentwickelten photographischen Hilfen und der «primitiven» Wandtafel zu überbrücken; er kann in der Tat weitgehend die Wandtafel ersetzen.» (n. n. 1983, 578)

Angesprochen wird in dem Artikel die Bequemlichkeit für die Lehrpersonen und die damit gegebenen Möglichkeiten einer Optimierung des Frontalunterrichts. Unter der Überschrift «Der Unterrichtende steht immer in Front zur Klasse», heisst es:

«Sofortige Reaktionen auf visuelle Informationen können vom Lehrer wahrgenommen werden und der lebendige stete Kontakt mit der Klasse wird beibehalten. Dieser stete Kontakt zeigt dem Lehrer auch die Momente deutlich an, wenn die Klasse einen etwas gelockerten Unterricht benötigt, um das psychologische Wohlbefinden aufrechtzuerhalten.» (ebd., 579)

Die mit den Overhead-Projektoren gegebene Innovationskraft scheint allerdings klein und war wie beschrieben vor allem auf eine frontalunterrichtliche Didaktik bezogen. Dennoch - oder gerade deswegen - setzten sich die einfach zu benutzenden Overhead-Projektoren als technische Lehrmittel durch - bis sie dann im digitalen Zeitalter von der Entwicklung von Visualizern und interaktiven Whiteboards, die in die digitalen Netzwerke integriert waren, abgelöst wurden.

Eine viel breitere Innovationskraft versprach ein zweiter grossangelegter Versuch einer Technologisierung des Unterrichts, nämlich der, auf einer kybernetisch-informationstheoretischen Didaktik aufbauende «Programmierte Unterricht». Dieser beruhte auf behavioristischen Lernkonzepten der Lernpsychologie und dem dort vertretenen Ansatz des 
«operanten Konditionierens». Angestrebt wurde eine Individualisierung des Lernens über die selbsttätige Rückkopplung der Lernenden. Auf diese Weise sollte sich ein kleinschrittiges Lernen an Lernzielen orientieren.

Über die Zerlegung der Inhalte in kleinste Teilaufgaben und darauf beruhendem Feedback sollte das Lernen abgesichert werden. Durch Drill und Practice und repetitives Üben sollte Verhalten verändert werden - wobei bereits hier mechanische Lernmaschinen eingesetzt wurden (vgl. Petko 2014, 27). In manchen Konzepten der damaligen Curriculumforschung sollte der behavioristische Lernzielansatz auf den gesamten Unterricht angewandt werden.

Dennoch erreichte der programmierte Unterricht mit seinen Lernmaschinen keinen Durchbruch in der Schule. Lediglich die Sprachlabore, die das audiovisuelle Lernen in den Unterricht einbringen wollten, wurden als Durchbruch der Technik gefeiert, blieben aber trotzdem in der schulischen Nutzung sehr weit zurück. Die stereotypen Muster des Lernens, die in diesem Lernmodell angelegt waren, verbreiteten eher Langeweile und Demotivierung, wenn sie häufig und routinemässig benutzt wurden.

Anne Bosche und Michael Geiss berichten über Versuche im schweizerischen Kanton Zürich mit Sprachlaboren, die Ende der 1960er Jahre mit grossem technischem Optimismus eingeführt wurden. Didaktisch wurde in der Folge bemängelt, dass die Schülerinnen und Schüler oft allein gelassen würden, wenn sich die Lehrpersonen bei den einzelnen Lernenden zuschalten. Der (traditionelle) einfache Unterricht in kleinen Gruppen könne die Defizite des bisherigen Sprachunterrichts bedeutend besser auffangen. Zudem wurden der hohe programmiertechnische Aufwand und die Reparaturanfälligkeit der Technik kritisiert:

«Doch auch diese Maßnahmen zeitigten nicht den gewünschten Erfolg. 1974 wurden auch in der Kommission für Sprachlabore Probleme mit ihrer Nutzung thematisiert. Technische Mängel bei Kopfhörern und der große finanzielle Aufwand wurden beanstandet und der Missbrauch der Anlagen durch die Schüler beklagt - von 〈Aggression〉 und 〈Sabotageakte(n)〉 war die Rede. Hans Weber von der Kantonsschule Solothurn, der als Gast einer Sitzung der Kommission beiwohnen durfte, äußerte seine massiven Zweifel an der Methode. Zur Behebung der technischen Mängel wurden in der Kommission 
nun der Einsatz und die Ausbildung von Feinmechanikern diskutiert. Das Sprachlabor war Mitte der 1970er Jahre endgültig in eine Legitimationskrise geraten: Sprachlabore würden zu wenig genutzt und wenn man sie nutze, dann 〈letztlich nur darum, weil man sie hat), hieß es in derselben Sitzung. Im gleichen Jahr wurde die Versuchsphase für beendet erklärt.» (Bosche und Geiss 2010, 134)

\subsection{Die ersten Rechner im Unterricht der Volksschulen}

Lernen mit Medien als zentraler Ansatz unterrichtlichen Handelns setzte sich im Sinne des programmierten Unterrichts also in den Schulen nicht durch. Ähnlich ging es im Übergang zur Digitalisierung dem Programmieren, das die informationstechnische Seite der Medien aufnahm und mit den ersten PCs zur Forderung führte, dass Programmieren zur zukünftigen Alphabetisierung gehöre - wie Rechnen, Lesen und Schreiben. Allerdings setzten sich diese Perspektiven in der Schule nicht durch schon weil die Lehrpersonen dadurch überfordert gewesen wären. Zudem schien es auszureichen, fit für die grafikorientierten Benutzeroberflächen zu werden, welche die Arbeit mit den Rechnern erleichterten.

Man setzte deshalb ab den 1980er Jahren auf eine «Alltagsinformatik», welche mit den Anwendungen praktisch arbeitete, die in die grafikorientierten Oberflächen der Rechner integriert werden konnten. Konzeptuell an den behavioristischen Ideen des programmierten Unterrichts und dessen individualisiertem Übungsansatz angelehnt kamen Übungen auf CDs hinzu, die von Software- und Lehrplanfirmen entwickelt wurden - bis hin zu Autorentools wie Hotpotatoes, mit denen Lehrpersonen einfache Abfrageübungen selbst erzeugen konnten. Übungen zum Lesen, zur Rechtschreibung oder zur Mathematik konnten so z. B. zur speziellen Förderung einzelner Schülerinnen und Schüler auf Diskette und später auf CD eingesetzt werden. Dazu kamen audiovisuelle Mittel der Medienpädagogik, die sich an die alte analoge Videoarbeit anschlossen - bis hin zu einigen besonders beliebten Einsatzmöglichkeiten wie «Stop-Trick», mit denen man gleichzeitig auch lernen konnte, wie Filmszenen sich aus Einzelbildern zusammensetzen. 
Mit der Entwicklung des Internets stellte sich zudem auch für die Schule die Frage, wie auf dieser Basis - etwa mit «Google» Informationen zu suchen sind. Eines der ersten didaktischen Modelle war damals das von Bernie Dodge (2001) entwickelte Modell der WebQuests. Allerdings war es nur eine Minderheit didaktisch an digitalen Medien interessierten Lehrpersonen, die solche Lernelemente auch nachhaltig in ihrem Unterricht einsetzten.

Mit der Digitalisierung rückte dann aber - z. T. wieder unter bewahrpädagogischer Perspektive - insbesondere das Lernen über Medien in den Mittelpunkt. Denn die neuen Medien (vom PC bis zu den Handys) waren für viele Eltern mit Unsicherheit verbunden - was sollte und durfte man Kindern und Jugendlichen zumuten, die als sog. «digital Natives» unbefangen und manchmal auch unkritisch mit solchen Geräten umgingen. Im Mittelpunkt standen Fragen wie diejenigen nach Computersüchten, Fragen nach vertrauenswürdigen Informationen vs. Fake-News, das Einrichten von sicheren persönlichen Profilen in den sozialen Medien (Facebook, Instagram), der Schutz vor sexuellem Missbrauch über Internet-Chatrooms etc. Ein Beispiel für diese Fundamentalkritik gibt Spitzer:

«Manche Netzaktivitäten wurden bei Internetsüchtigen besonders häufig nachgewiesen: Das Einkaufen im Internet, exzessiver Videokonsum, die intensive Nutzung von sozialen Online- Netzwerken, Chatrooms und Online-Spielen sowie die intensive nächtliche Internetnutzung. Man kann sich also kaum des Eindrucks erwehren, dass Studenten, die vor allem im Netz unterwegs sind, ihre Aktivitäten in der realen Welt reduzieren und damit sozial zunehmend isoliert sind» (Spitzer 2012, 267)

Solche Fragestellungen liessen sich mit dem Arsenal traditioneller Bewahrpädagogik beantworten. An vielen Schulen wurde sich nicht mit der Thematik der Digitalisierung auseinandergesetzt, weil nur wenige Lehrpersonen das Grundwissen erworben hatten, um Fragen des digitalen Umgangs mit Medien im Unterricht zu behandeln. Noch bis vor kurzem war an vielen Orten das Lernen mit digitalen Medien auch deshalb eingeschränkt, weil die Skepsis der Schulen und Schulbehörden gegenüber den Medien im 
Vordergrund stand, indem sie schlicht Handyverbote auferlegten oder die Nutzung des Rechners im Unterricht nur für enge - von den Lehrpersonen kontrollierte - Unterrichtsaufgaben zuliessen.

\subsection{Lernen im privaten Bereich}

Ganz anders sah es im privaten Bereich aus. Schon bei den Hausaufgaben waren der Rechner oder das Handy zuhause ein ständiger Begleiter - mit dem man sich unter Kolleginnen und Kollegen austauschte. Für Aufsätze und Vorträge recherchieren die Schülerinnen und Schüler ganz selbstverständlich im Internet. Besonders beliebt als Lernhilfe sind Erklärvideos, indem Schülerinnen und Schüler einander Lernschritte per Videos erklären und ihren Mitschülern zugänglich machen. Im Netz gibt es geradezu Profis für Erklärvideos, die massenweise solche Videos produzieren, auf Videoportalen zugänglich machen und damit letztlich mit Lehrerinnen und Lehrern konkurrieren. Denn häufig geben Schülerinnen und Schüler an, von Peers die wesentlichen Aspekte einer Lernaufgabe leichter zu lernen als in der Schule, wo dieselbe Aufgabe im Frontalunterricht vor 20 und mehr Schülerinnen und Schüler an der Wandtafel erklärt wurde.

So betont Frank Schlegel:

«Egal ob Deutsch, Kunst, Sprachen, Gesellschafts- oder MINT-Fach - Erklärvideos decken Themen aus allen Fächern ab, von den Grundlagen bis hin zum Spezialwissen. Aus der hohen Alltagsrelevanz des Erklärvideos und der Möglichkeit, solche Videos mit vergleichsweise geringem Aufwand zu rezipieren und selbst zu produzieren, ergeben sich neue Chancen für die Unterrichtsgestaltung» (Schlegel 2016).

Allerdings sind Erklärvideos umstritten geblieben. So heisst es 2019 auf der Website des Landesbildungsservers Baden-Württemberg:

«Jeder Schüler kennt Lernvideos bereits aus dem Unterricht oder der privaten Nutzung. Es gibt viele hochwertige Erklärvideos, die Schülerinnen und Schülern beim Lernen unterstützen, aber auch massiv vereinfachende Videos, in denen falsche Fakten wiedergegeben werden oder Videos, die schlecht produziert sind»(Institut für Bildungsanalysen Baden-Württemberg - IBBW, o. J.). 
Vor allem aber können Erklärvideos auch verwirren, wenn sie - z. B. in der Mathematik - alternative Bearbeitungsregeln vorschlagen, die mit dem Lernansatz der Lehrpersonen im Widerspruch stehen und dabei die Unsicherheit der betroffenen Schülerinnen und Schüler noch verstärken.

\subsection{Der Rechnereinsatz an den heutigen Schulen}

Zusammenfassend ist aus aktueller Sicht festzustellen, dass Medien heute zwar häufiger eingesetzt werden, aber kaum zu wirklichen Unterrichtsinnovationen geführt haben. Auch technische Geräte wie die Whiteboards erschienen den meisten Lehrpersonen als zu komplex und wurden häufig nur wenig genutzt. Es gab wenige Nerds unter den Lehrpersonen, die sich intensiv einarbeiteten und eine Mehrheit, die sich der Arbeit mit solchen Geräten entzogen.

Obwohl in Deutschland die Zahl der installierten interaktiven Whiteboards zugenommen hat, unterstreichen diese Situation Zahlen, wie sie Irion berichtet:

«Interaktive Whiteboards (IWBs) fanden in Deutschland im Rahmen schulischer Lehr-Lernsettings bislang nur vereinzelt Verwendung. Im Gegensatz zu Großbritannien, wo gross angelegte Förderprogramme in zweistelliger Millionenhöhe schon 2002 entwickelt wurden, konnte in Deutschland für die Einführung digitaler, interaktiver Tafeln im Klassenzimmer bislang nur auf punktuelle Förderprogramme oder vereinzelte Schulträger-Initiativen zurückgegriffen werden.» (Irion 2012, 175).

Letztlich wiederholt sich hier Ähnliches wie beim programmierten Lernen der 1980er Jahre, indem innovative Techniken durch ihre Komplexität nicht zum Tragen kamen und an technischen Schwierigkeiten scheiterten.

Seit in der Schweiz der Lehrplan2l eingeführt wurde, hat sich auch die Diskussion um die Entwicklung von Lehrmitteln im Zeitalter der Digitalisierung verstärkt (vgl. z. B. Döbeli Honegger, Hielscher, und Hartmann 2018). Nach Bezzola (2019) soll man die künftige Vision der Lehrmittel mit «plattformunabhängigen, adaptiven Lernmaterialien» beschrieben 
können. Lehrmittel sollen verstärkt zu offenen und flexiblen Produkten werden, deren roter Faden - mindestens in den Vorstellungen der Lehrmittelverlage nach wie vor das Print-Lehrmittel ist. Vor allem geht es um die Erweiterung und Anreicherung der Schulbücher mit digitalen Komponenten. Für die Verlage handelt es sich hier oft noch um einen Spagat: Die sehr unterschiedliche Ausrüstung mit digitalen Mittel führt von 1:1 Relation im Verhältnis von Schülerinnen und Schülern/Computer bis zu sehr sporadisch genutzten Geräten pro Klassenzimmer (vgl. Bezzola 2019, 17). Das bremst die Entwicklung digitaler Materialien, weil es möglich sein muss, die vorgegebenen Lernziele der Curricula in allen Settings gleichermassen zu erreichen, also auch dort, wo keine oder nur wenige Rechner zur Verfügung stehen. Es darf also kein digitales Material existieren, das solche Lernziele allein realisiert und keine Alternativen auf herkömmlicher Basis vorsieht. Jedenfalls ist jene Utopie, wonach im Mittelpunkt des Unterrichts je nach Fach und Aufgabe auch digitale Materialien (Internet, Social Media etc.) als alleinstehendes Lernmaterial stehen können, trotz aller vielgelobter Digitaloffensiven von der Realisierung noch weit entfernt.

Insgesamt ergibt sich damit ein Bild, wonach digitale Rechner bis in die Gegenwart in der Volksschule ergänzend und nicht als zentrale Lehr- und Lernmittel benutzt wurden, zumal sich auch die Lehrpersonen oft durch die neuen Technologien überfordert fühlten. Trotz staatlicher Initiativen wie dem Lehrplan 21 in der Schweiz ist festzustellen, dass man nach wie vor von einer Übergangszeit der Digitalisierung sprechen muss, in welcher Fortschritte nur langsam zu erzielen sind. Auch wenn Hochschulen und Universitäten beginnen, die Medienbildung mit obligatorischen Semesterwochenstunden ins Programm aufzunehmen, so ist der Umfang für eine grundlegende und vertiefte Ausbildung, die auch informatische Grundbestandteile enthält, viel zu eng.

Dies bestätigt meines Erachtens auch der JAMESfocus-Bericht «Digitale Medien im Unterricht» von 2019 für die Schweiz, für welchen über 100 Schweizer Lehrkräfte auf der Sekundarstufe zum Einsatz digitaler Medien im Unterricht befragt wurden. Generell stehen diese den digitalen Medien positiv gegenüber und setzen sie im Unterricht häufig ein: 
«90\% der Lehrpersonen geben an, dass sie häufig oder sehr häufig im Internet recherchieren. Drei Viertel geben an, häufig Inhalte mithilfe digitaler Medien zu präsentieren. Rund die Hälfte der Lehrpersonen gibt an, dass sie häufig zeigen/erklären, wie man etwas an einem digitalen Gerät (z. B. Computer, Smartphone, Tablet) mache.» (Suter u. a. 2019, 9 ff.)

Daneben werden auch digitale und audiovisuelle Inhalte genutzt (Video-Clips von YouTube, Fernsehsendungen und Online-Texte etc.). Trotzdem zeigen auch diese Daten, dass digitale Medien thematisch nicht im Zentrum des offiziellen Lehrplans stehen, sondern zur Anreicherung und Erweiterung der Unterrichtsmöglichkeiten genutzt werden.

Positiv ist immerhin festzuhalten, dass die grundlegende Skepsis an den digitalen Medien und damit die Totalverweigerung abgenommen hat. Allerdings stellt das Unterrichten mit digitalen Medien für manche Lehrpersonen grosse oder zu grosse Anforderungen - und sie sind hilflos darin, wie sie Kompetenzen in diesem Bereich vermitteln können. So fürchten sie, nicht über das nötige Know-how zu verfügen, bzw. sich dieses nur schwer aneignen zu können. So kommentiert Michael In Albon den JAMESfocus Bericht im Swisscom-Magazin.

«Die wichtigste Kritik aus dem Bericht betrifft aber die digitale Fitness der Lehrpersonen. Sie wurden befragt, wo sie sich Unterstützung wünschen oder nötig haben. Dabei zeigt sich ein Bild der Unsicherheit. Ein Drittel der Lehrpersonen fühlen sich nicht fit in technischen Kompetenzen, über ein Drittel ist sich der sozialen und gesellschaftlichen Wirkungen von Medien und Medieninhalten nicht sicher, und fast zwei Drittel wissen nicht, was genau denn im Unterricht zum Einsatz kommen soll.» (In Albon 2019)

\section{Lernen mit digitalen Medien - ein Zwischenfazit}

Was an der heutigen Situation der Schulen auffällt, sind drei Aspekte:

1. Lehrpersonen nutzen eher einfachere Anwendungsformen der digitalen Medien und sie fühlen sich oft noch unsicher, wenn es um das Know-how geht, das sie besitzen. Es dürfte auch nicht so einfach sein, 
die bestehenden Defizite bei Lehrpersonen, die schon längere Zeit im Schuldienst stehen, aufzufangen. Geht man davon aus, dass eine fundierte Weiterbildung kaum weniger als 2 Semesterwochenstunden über rund 3-4 Semester bedarf, dann ist es kaum möglich, solche Lücken über temporäre Weiterbildungsmassnahmen kurzfristig zu schliessen. Zwar werden zunehmend gut ausgebildete Lehrkräfte neu in den Schuldienst eintreten. Es dürfte aber sicher noch fünf bis zehn Jahre dauern, bis die Schulen von einer Population von Lehrpersonen ausgehen können, die für den digitalen Wandel adäquat gerüstet und fit ist. Überall dort, wo in der Grundausbildung noch weniger Angebote bestehen, dürfte der Nachholbedarf noch weit grösser sein.

2. Konzeptionell ist es weiterhin eine externe Sicht auf Medien, die in den Schulen vorherrschend ist. Medien sind danach Geräte, an die man sich ankoppelt, wenn man sie für Unterrichtszwecke braucht, oder die als Inhalt aus dem Alltag eines Kommentars bedürfen. Zu wenig deutlich wird dabei, dass Medien immer häufiger integral und alternativlos in den Alltag eingebunden sind, weil ohne ihren Einbezug keine Bildung mehr möglich ist. War Fahrplanlesen noch vor wenigen Jahren ein Inhalt, der auf Papierbasis gelernt werden konnte, gibt es heute fast nur noch Online-Fahrpläne. Und diese sind mit vielen neuen Funktionen angereichert, die ohne Rechner nicht mehr in den Blick kommen. Auch die Informationssuche über das Internet (etwa: über Wikipedia und Browser-Abfragen) ist zwingend, seit die Konversationslexika in ihrer Entwicklung eingestellt wurden. Dies ist aber nur der Anfang. Dienstleistungsunternehmen aller Couleur dünnen ihre Schalterdienste aus. Bald wird man Leistungen wie Post, Bankgeschäfte oder Ticketkauf nur noch digital vornehmen können. Und wenn es auch in handwerklichen Berufen immer selbstverständlicher ist, dass grosse Teile der früheren händischen Fähigkeiten an die Steuerung über Maschinen abgegeben werden, wird auch dort das Lernen wesentlich mit dem Erlernen von digitalen Kompetenzen verbunden sein.

Dasselbe gilt für das Leben mit technischen Werkzeugen wie dem Handy im heutigen digitalen Alltag. Es ist deshalb von vorneherein die falsche Frage, wenn man sich überlegt, wie man wenigstens für die 
Unterrichtszeit, die Handys aus dem Unterricht verbannen kann. Auch wenn die Telefone unerwünschte Aktivitäten zur Folge haben können, die dem «offiziellen» Unterricht entgegenstehen, ist viel eher zu fragen, wie solche Geräte als positive Ressourcen in das Lernen an den Schulen einbezogen werden können. Auch im Arbeitsalltag der Erwachsenen wird ja nicht versucht, die digitalen Geräte über Verbote von den Mitarbeitenden fern zu halten, sondern man sucht die Ressourcen in den Arbeitsalltag einzubeziehen, die mit den Medien verbunden sind. So ist es selbstverständlich, dass die digital vernetzte Arbeitswelt ein Teil des täglichen Arbeitsprozesses geworden ist.

Genau dies ist eine Aufgabe, die noch viel nachhaltiger von den Schulen aufzugreifen ist: Wie kann der digitale Alltag in den Schulen abgebildet und fachübergreifend ins alltägliche Lernen einbezogen werden - auch dort, wo keine Überschrift «Medienbildung» die Inhalte der Wissensdomäne des digitalen Lernens zuweist?

3. Mit der rasant zunehmenden Digitalisierung ist in letzter Zeit die Diskussion um die Informatik neu aufgeflammt. Vor allem in Wirtschaftskreisen wird in den Informatikkenntnissen jene Wissensdomäne gesehen, welche für das Innovationspotential der digitalen Gesellschaft zentral ist. Dies schliesst letztlich an die Diskussionen in den 1980er Jahren des letzten Jahrhunderts an, wo in der Mediendiskussion das Programmieren und Steuern von Computern als wichtiger Bestandteil des fachlichen Diskurses gesehen wurde: Man denke nur an die Überlegungen Paperts (1982) zur Computersprache «Logo», die damals aktiv verbreitet wurden. Allerdings setzten sich weder Logo noch das Programmieren breit in den Schulen durch; es schien medienpädagogisch wichtiger zu sein, die Rechner im Sinne einer anwendungsorientierten Alltagsinformatik bedienen zu lernen, während die komplexeren Fragen der informatischen Grundlagen sehr rasch wieder in den Hintergrund traten. Dieser pragmatische Strang einer digitalen Medienbildung verstärkte sich noch mit dem Internet und den sozialen Medien, deren Beherrschung nur wenig mit algorithmischem Wissen und Programmieren zu tun hat. 


\section{Coding, Computational Thinking und konstruktivistische Lernansätze}

Am Schluss dieses Beitrags sollen dennoch einige Überlegungen zur Diskussion über die Informatik stehen - nicht zuletzt, weil die Digitalisierung alle Bereiche der Gesellschaft zu verändern beginnt und damit die Frage nach der Informatik wieder eines der vordringlichen Diskursthemen der Medienpädagogik geworden ist. Es wäre denn auch fatal, wenn eine Schule ohne Informatik mit dazu beitrüge, die sich andeutende digitale Revolution zu verschlafen.

Allerdings sind die Hoffnungen in die Informatik als neue Grundfertigkeit differenziert zu betrachten. Die erste Informatikwelle, die in den 1980er Jahren auf die Gesellschaft traf, hat durchaus zwiespältige Gefühle hinterlassen. Wer sich damals zum Programmierer ausbildete, musste erfahren, dass sich die Programmiersprachen schnell veränderten und neuere Technologien - etwa die ganze Netzwerktechnik - Anforderungen stellten, für welche viele Programmierer der ersten Stunde nicht ausgebildet waren, so dass sie aus dem Arbeitsmarkt ausschieden.

Allein auf Coding zu setzen, also auf das Erlernen einer Computersprache und deren Codes - erschiene mir auch heute noch kurzsichtig. Buckingham hat dies anhand englischer Erfahrungen differenziert beschrieben (vgl. Moser 2019, 241), wobei er skeptische Schlüsse zieht. Auf seiner Website fasst er Argumente zusammen, welche gegen das Programmieren sprechen. Einmal unterscheidet er zwischen Programmieren und der Computerwissenschaft. Beim reinen Programmieren handle es sich eher um eine niedrige Fähigkeit, die stark mit Routinen verbunden sei. So meint Buckingham (2016) in seinem Blog-Beitrag sarkastisch:

«If the government wants the UK to become a leading player in the global technology business, it may be much more in need of creative entrepreneurs than programming drones.»

Die Präzision und Klarheit der Computersprache mag zwar geeignet sein, Routinen zu entwickeln und analytisch Prozesse aufzudröseln. Es ist jedoch das Gegenteil jener Kreativität, die Buckingham für technologische Bildung einfordert. Kognitivistische und konstruktivistische Lernansätze, die Problemlösen und Eigenaktivität in den Vordergrund stellen, 
werden in traditionellen Coding-Konzepten oft vernachlässigt. Buckingham bestreitet zudem Thesen, wonach durch informatische Grundlagen gleichzeitig unsere logischen Denkfähigkeiten generell gefördert werden, um gesellschaftliche Probleme analytisch und logisch zu lösen. Ähnliche Behauptungen würden oft auch für das Erlernen von Schach oder Latein aufgestellt:

«Yet there is no convincing evidence that learning computer programming enables children to develop more general problem-solving skills, let alone that it will 'teach you how to think', as its advocates claim.» (Buckingham 2016)

Coding als formelles Lernen von Regeln und Algorithmen der Steuerung von Prozessen hat denn auch wenig mit kognitivistischem und konstruktivistischen Lernansätzen zu tun. Jeanette Wing (2008) setzt demgegenüber auf das von ihr geprägte Konzept eines «Computational Thinking», das sie wie folgt definiert: «Computational Thinking ist der Gedankenprozess, der sowohl die Formulierung eines Problems als auch die Repräsentation der Problemlösung so darstellt, dass sie von Menschen oder durch Maschinen ausgeführt werden könnte.» (zit. nach Lobe 2017). Das müssen nicht nur informatische Konzepte sein, auch das Backen eines Kuchens kann in der Form der Regeln eines Algorithmus beschrieben werden (Repenning 2015, 6).

Allerdings geht es beim Computational Thinking stark um die direkte Anwendung von Konzepten aus der Informatik auf den Alltag. Dies kann durch den dabei gegebenen Grad der Anschaulichkeit das Verständnis von Kindern und Jugendlichen zwar erleichtern. Dennoch wäre zu fragen, um beim Beispiel des Kuchenbackens zu bleiben, ob es genügt, lediglich entsprechende Routinen zu lernen, um das Backen zu standardisieren und erfolgreich zu machen. Was zu wenig zum Ausdruck kommt, ist das Experimentieren und Ausprobieren, welches über die Anwendung von algorithmischen Regeln hinausgeht und zu Aktivitäten führt, innerhalb derer es um die Auseinandersetzung mit eigenständigem Wissen geht - also zu Lernkonzepten, wo in Projekten Wissen konstruiert und eigene Bedeutungen und Lernerfahrungen in den Themen und Inhalten der Digitalität entdeckt werden (vgl. Moser 2008, 58). 
In der Diskussion um die Digitalisierung im schulischen Raum begründet Doebeli Honegger (2017) die Notwendigkeit der Vermittlung von Informatikkenntnissen damit, dass Informatik und Computer einen virtuellen Raum eröffnen, in welchem sich Konzepte modellieren und simulieren liessen. Die Informatik stelle dazu Werkzeuge zur theoretischen Beschreibung von Strukturen und Abläufen bereit, wie auch konkrete Systeme in Form von Computern, um diese Strukturen und Abläufe zu implementieren und zu simulieren. So liessen sich sowohl die physikalischen Gesetze unserer Erde nachbauen wie auch Welten simulieren, die nach ganz anderen physikalischen Gesetzen funktionierten.

Für das digitale Lernen scheint mir in diesem Zusammenhang der Begriff der Simulation von Welten besonders wichtig, erscheint doch in vielen bestehenden Informatikmodellen die damit angedeutete kreative und selbstgestaltende Seite einer digitalen Bildung oft nur in zweiter Linie. Es ist zwar richtig, wenn die «Charta für Digitale Bildung» von 2019 festhält, dass alle Schülerinnen und Schüler schon in der allgemeinbildenden Schule die Phänomene, Gegenstände und Prozesse der durch Digitalisierung geprägten Welt aus (1) technischer, (2) gesellschaftlich-kultureller und (3) anwendungsbezogener Perspektive systematisch reflektieren, ergründen und gestalten lernen sollen, wobei auch die «Befähigung zur aktiven Mitgestaltung» eingeschlossen wird.

Problematisch wird dann aber die an dem Dagstuhl-Modell orientierte Grafik, die mitgeliefert wird:

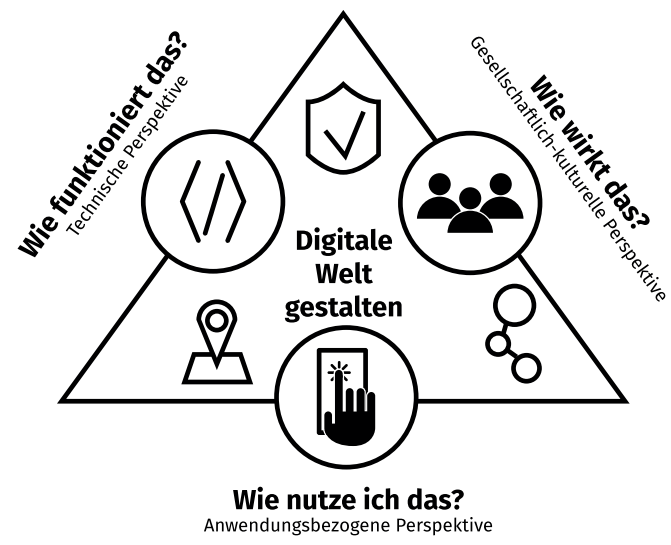

Abb. 1.: Charta für digitale Bildung (Quelle: https:/charta-digitale-bildung.de/ media/chart.svg) 
An der Grafik wird deutlich, was die Autorinnen und Autoren unter dem Aspekt der Gestaltung digitaler Welt verstehen, nämlich die Beantwortung der Fragen:

- Wie funktioniert das? (technisch)

- Wie wirkt das? (gesellschaftlich-kulturell)

- Wie nutze ich das (anwendungsbezogen).

Die Position der Lernenden ist dabei gemäss dieser Grafik rezeptiv festgelegt; sie müssen nachvollziehen, wie sich digitale Prozesse vollziehen, welche Folgen sie haben, und wie sie sich auf die Menschen auswirken. Routinen und Algorithmen sind dabei der systematische Rahmen, welcher die Handlungsspielräume der Lernenden vorgibt und einschränkt. Wenn man stärker konstruktivistisch argumentiert, dann werden hier die vorgegebenen Rahmen als «objektive Parameter» der Welt und ihres systematischen Aufbaus zu stark betont. Vielmehr ginge es in solchen Lernkonzepten darum, dass wir Weltentwürfe und Wissensstrukturen selbst «autopoietisch» konstruieren und gestalten. Während die Konzepte der Informatik über die dabei entwickelten Regeln routinemässige Abläufe zu entwickeln versuchen, sind nach konstruktivistischem Duktus Systeme operativ geschlossen und reagieren auf Perturbationen bzw. Verstörungen aus der Umwelt nach eigenen und von aussen nicht vorhersehbaren Regeln. Psychische Systeme können eben nicht belehrt werden, sondern es können lediglich Anstösse und Anregungen gegeben werden, um daraus besser zu verstehen, wie das «fremde» System diese verarbeitet.

\section{Medienpädagogische Akzentuierungen mit einem «kultur-technischen Werkunterricht»}

Medienkritisch gesprochen legt die Charta die Position der Lernenden zu stark auf den Nachvollzug der Digitalisierung fest und weniger auf die kreative Mit- und Weiterarbeit an einer digitalen Gesellschaft, die erst im Entstehen begriffen ist. Wenn es nicht einfach um wirtschaftskonforme Anpassung an diese geht, dann müssten viel stärker das eigene Gestalten und die kritische Auseinandersetzung mit der digitalen Gesellschaft in den Mittelpunkt von Lernprozessen kommen. Am ehesten können solche 
Ansprüche dort realisiert werden, wo digitale Lernprozesse nicht einfach Coding betreffen, sondern als digitale Projekte angelegt sind, in welchen das Experimentieren und Ausprobieren im Mittelpunkt stehen.

Hier wäre vorab die Makerspace-Bewegung zu nennen, die aus der DIY (Do It Yourself)-Bewegung entstanden ist, immer mehr aber traditionelles Handwerken mit Informatik verbindet. In diesem Zusammenhang ist auch das Arbeiten mit Kleinstcomputern wie Arduino zu nennen, mit denen technische Grundlagen in engem Zusammenhang mit praktischer Projektarbeit bearbeitet werden können. Das «Arduino Projektbuch» verdeutlicht die Richtung: «Arduino macht es so einfach wie möglich, winzige Computer, sogenanntes Microcontroller, zu programmieren, um Objekte interaktiv zu machen» (Smart Projects 2017, 5). Das, was im Allgemeinen im digitalen Alltag unbemerkt bleibt, wird hier in Arbeitsvorhaben integriert, die über Sensoren und Aktoren erfasst und gesteuert werden. Ziel ist es: «Dank Arduino lernen Designer, Künstler, Bastler und Studenten jeden Alters, Dinge zu erschaffen, die aufleuchten, sich bewegen und mit Menschen, Tieren, Pflanzen und dem Rest der Welt interagieren» (ebd., 4).

Digitalisierung bedeutet denn auch nicht, formelles Coding als Lehrbuchwissen zu lernen. Wichtig ist hier vielmehr das Experimentieren in Räumen, wo man digitale Projekte durchführen kann, die auf alltägliche Erfahrungen zurückgreifen - etwa wie die Makerspace Räume. Es sind sozusagen «third spaces», welche digitales Arbeiten, und Reflexion, Technik und Kultur miteinander verbinden. Potter und Mc Dougall $(2017,37)$ sehen darin neutrale Plätze - sozusagen «Übergangsräume» zwischen formellem Lernen und ausserschulischem Alltag, die geeignet sind, kreatives Arbeiten herauszufordern, im dadurch zwischen kulturellen und technischen Aspekten Verbindungen zu erzeugen. Die Arbeit mit dem Soziomateriellen soll das Virtuelle mit dem Materiellen verknüpfen, um dabei konkrete Produkte und deren Reflexion miteinander zu verbinden (vgl. Moser 2019, 246).

Es geht also nicht darum, für Informatik und Medienbildung in den Schulen ein neues theoretisches Fach «Informatik» zu schaffen. Vielmehr wäre eine neue Form des Werkunterrichts zu entwickeln, der praktisch orientiert ist und Theorie und Praxis, Handwerk und digitalisierte Praxis, experimentelles Handeln, Reflexion und eigenständiges Lernen miteinander 
verbindet. Dieser neue Werkunterricht sollte als integrierendes Fach an einem Halbtag pro Woche das Digitale und das Handwerkliche mit dem Alltäglichen so verknüpfen, dass die neue Kultur der digitalen Gesellschaft mit allen ihren Facetten - positiven und negativen - bearbeitet und experimentell angeeignet werden kann.

Die Interaktion des Digitalen mit dem Soziokulturellen ist dabei auch Ausgangspunkt des medienkritischen Reflektierens, das häufig noch zu wenig Teil des Unterrichts ist. Dazu gehören etwa Internetrecherchen zu Memes, Twitter, Fake-News, Big Data etc. Für die damit verbundenen gesellschaftlichen Phänomene eines zunehmenden digitalen Kapitalismus hat Horst Niesyto u. a. folgende Problemfelder beschrieben (Niesyto 2018, 62):

- Die kommerzielle Enteignung und Ausbeutung persönlicher Datenprofile und der damit verknüpfte Kommerzialisierungsschub;

- Das Entstehen neuartiger, teilweise totalitärer Machtstrukturen in Verbindung mit Big Data und damit verbunden neuer gesellschaftlicher Kontrolle;

- Die verstärkte Entwicklung körperlich-digitaler Mensch-ComputerSchnittstellen in Richtung eines homo digitalis.

Gerade im Bereich der Digitalisierung wachsen eine medienkritische Medienpädagogik und die Informatik zusammen, da eine kompetente $\mathrm{Me}-$ dienkritik nicht ohne informatisches Grundwissen auskommt:

- Wer beurteilen will, wie gefährlich Big Data ist, muss auch etwas über die dahinterstehenden medientechnischen Voraussetzungen wissen.

- Eine kritische Medienkompetenz ist auch wichtig, wenn man die Entwicklung der Smart Homes und weiterer Smartsysteme verfolgt. Sind z. B. Geräte wie Alexa sicher, oder muss man annehmen, dass sie als Datensensoren dessen Anbieter Amazon eine Unmenge von Daten liefern? Wie weit sind Smartphones nicht nur Fotoapparate für meinen Privatgebrauch, sondern Datenschleudern, die uns als gläserne Wesen transparent machen? 
Die Diskussion solcher Themen in der Schule ist leicht möglich, wenn man in den Zeitungen und Zeitschriften und im Internet entsprechende Diskussionen verfolgt und diskutiert: Themen könnten z. B. sein, wie sinnvoll E-Trottinetts als alternatives Verkehrsmittel sind, wie ausbeuterisch in diesem Zusammenhang der Plattformkapitalismus sein kann, wie die Senioren aus dem gesellschaftlichen Alltag ausgegliedert werden, wenn immer mehr Dienste, die sie benötigen, nur noch über das Internet erreichbar sind. Dabei ist es wichtig, dass das eigene digitale Know-how nicht allein für medienkritische Reflexionen genutzt wird, sondern dass auch versucht wird, über die eigenen digitalen Kompetenzen bei der Entwicklung und Diskussion von Lösungen mitzuhelfen.

Zur Formulierung von Medienkompetenz für ein digitales Zeitalter hat Dieter Baacke am Ende des analogen Zeitalters bereits 1997 eine provokative Formulierung gefunden, die man als visionäre Überschrift über die Medienpädagogik des 21. Jahrhunderts setzen könnte: «Die Freibeuter auf den Informationsmeeren sind heute die Hacker. Nicht mehr Besitz und Produktion, sondern die Teilhabe an den Flüssen der Information, das gilt heute» (Baacke 1997, 101). Die Teilhabe an den third spaces bedeutet denn auch, nicht einfach angepasste und funktionale Projekte zu gestalten. Medienkritisch wäre beizufügen, dass digitales Lernen auch bedeutet, sich als Freibeuter der digitalen Informationsgesellschaft um die eigenen Freiräume in dieser Gesellschaft zu kümmern und die Mitgestaltung an der entstehenden digitalen Gesellschaft einzufordern.

\section{Literatur}

Baacke, Dieter. 1997. Medienpädagogik. Nachdr. Grundlagen der Medienkommunikation 1. Tübingen: Niemeyer.

Beer, Ulrich. 1960. Geheime Miterzieher der Jugend: Macht u. Wirkung d. Massenmedien. 8., Völlig neu bearb. u. erw. Aufl. Jugend, Bildung, Erziehung. Tübingen: Katzmann.

Bezzola, Patric. 2019. «Vision und Realität - Lehrmittelentwicklung in Zeiten der Digitalisierung». profil. Das Magazin für Lehren und Lernen, Nr. 2: 16-17. https:// www.profil-online.ch/profil-online-media/docs/2019/SVplus_profil_2019-02_ web.pdf. 
Bosche, Anne, und Michael Geiss. 2010. «Das Sprachlabor - Steuerung und Sabotage eines Unterrichtsmittels». In Jahrbuch für historische Bildungsforschung, herausgegeben von Carola Groppe, Klaus-Peter Horn, und Gerhard Kluchert, 16:119-39. Bad Heilbrunn: Verlag Julius Klinkhardt. http://nbn-resolving.org/ urn:nbn:de:0111-pedocs-145838.

Buckingham, David. 2016. «Why children should NOT be taught to code». 2016. https://davidbuckingham.net/2015/07/13/why-children-should-not-be-taughtto-codel.

Döbeli Honegger, Beat. 2017. Mehr als O und 1: Schule in einer digitalisierten Welt. 2., Durchgesehene Auflage. Bern: hep, der Bildungsverlag.

Döbeli Honegger, Beat, Michael Hielscher, und Werner Hartmann. 2018. «Lehrmittel in einer digitalen Welt». Interkantonale Lehrmittelzentrale ilz. https:// ilz.ch/wp-content/uploads/2020/06/181112-51h7-Expertenbericht-Lehrmittelin-einer-digitalen-Welt.pdf.

Dodge, Bernie. 2001. «FOCUS: Five rules for writing great WebQuests». Learning \& Leading with Technology 28 (8): 6-9. http://webquest.org/sdsu/focus/focus. pdf.

In Albon, Michael. 2019. "Medien im Unterricht: Lehrer müssen nachsitzen». Swisscom Magazin, 13. August 2019, Abschn. Digitalisierung im Alltag. https://www.swisscom.ch/de/magazin/digitalisierung-im-alltag/medien-im-unterricht-lehrer-muessen-nachsitzen/.

Institut für Bildungsanalysen Baden-Württemberg - IBBW. o. J. «Bewertung und Produktion von Erklärvideos». Landesbildungsserver Baden-Württemberg. https://www.schule-bw.de/faecher-und-schularten/sprachen-und-literatur/ deutsch/unterrichtseinheiten/projekte/erklaervideo-deutsch.

Irion, Thomas. 2012. «Interaktive Whiteboards im Grundschulunterricht. Didaktische Herausforderungen für die Nutzung digitaler Tafeln bei der Gestaltung von Lernumgebungen in der Primarstufe». In Jahrbuch Medienpädagogik 9, herausgegeben von Renate Schulz-Zander, Birgit Eickelmann, Heinz Moser, Horst Niesyto, und Petra Grell, 175-96. Wiesbaden: VS Verlag für Sozialwissenschaften. https://doi.org/10.1007/978-3-531-94219-3_9.

Lobe, Adrian. 2017. «Nicht nachdenken, programmieren!» NZZ - Neue Zürcher Zeitung, 12. Mai 2017, Abschn. Feuilleton. https://www.nzz.ch/feuilleton/soll-dermensch-wie-ein-computer-denken-ld.1292090.

Moser, Heinz. 2008. Einführung in die Netzdidaktik: Lehren und Lernen in der Wissensgesellschaft. Baltmannsweiler: Schneider-Verl. Hohengehren.

Moser, Heinz. 2019. Einführung in die Medienpädagogik: Aufwachsen im digitalen Zeitalter. 6., Überarbeitete und Aktualisierte Auflage 2019. Wiesbaden: Springer Fachmedien Wiesbaden GmbH. https://doi.org/10.1007/978-3-658-232085 .

n. n. 1983. «Der Unterricht mit dem Hellraumprojektor». schweizer schule, Nr. 12: 578-82. https://doi.org/10.5169/SEALS-534925. 
Niesyto, Horst. 2018. «Medienkritik - Entwicklungslinien und aktuelle Herausforderungen». In Medienkritik im digitalen Zeitalter, herausgegeben von Horst Niesyto und Heinz Moser, 59-77. Medienpädagogik interdisziplinär 11. München: kopaed.

Papert, Seymour. 1982. Mindstorms: Kinder, Computer und Neues Lernen. Basel: Birkhäuser. https://doi.org/10.1007/978-3-0348-5357-6.

Petko, Dominik. 2014. Einführung in die Mediendidaktik: Lehren und Lernen mit digitalen Medien. Bildungswissen Lehramt 25. Weinheim: Beltz.

Potter, John, und Julian McDougall. 2017. Digital media, culture and education: theorising third space literacies. London: Palgrave Macmillan.

Repenning, Alexander. 2015. "Computational Thinking in der Lehrerbildung». Hasler Stiftung, Bern. https://haslerstiftung.ch/wp-content/uploads/ documents/d/fit_schriftenreihe/haslerstiftung_schriften04_de_v02.pdf.

Rummler, Klaus, Ilka Koppel, Sandra Aßmann, Patrick Bettinger, und Karsten D. Wolf, Hrsg. 2020. Jahrbuch Medienpädagogik 17: Lernen mit und über Medien in einer digitalen Welt. Bd. 17. Jahrbuch Medienpädagogik. Zürich: Zeitschrift MedienPädagogik / Sektion Medienpädagogik (DGfE). https://doi.org/10.21240/ mpaed/jb17.X.

Schlegel, Frank. 2016. "Erklärvideos im Unterricht. Einstieg in die Filmbildung mit YouTube-Formaten - Workshop für Lehrkräfte und MedienberaterInnen». FILM+SCHULE NRW, LWL-Medienzentrum für Westfalen. https://medienkompetenzrahmen.nrw/fileadmin/dokumente/user_upload/ Erkl\%C3\%A4rvideos-im-Unterricht.pdf.

Smart Projects. 2017. Arduino Starter Kit. Haar: Franzis.

Spitzer, Manfred. 2012. Digitale Demenz: wie wir uns und unsere Kinder um den Verstand bringen. München: Droemer.

Suter, Lilian, Céline Külling, Jael Bernath, Gregor Waller, Isabel Willemse, und Daniel Süss. 2019. «JAMESfocus. Digitale Medien im Unterricht». Zürcher Hochschule für angewandte Wissenschaften, Fachgruppe Medienpsychologie. https://www.zhaw.ch/storage/psychologie/upload/forschung/medienpsychologie/james/jamesfocus/2019/JAMESfocus_Digitale_Medien_im_Unterricht_de.pdf.

Wing, Jeannette M. 2008. «Computational Thinking and Thinking about Computing». Philosophical Transactions of the Royal Society A: Mathematical, Physical and Engineering Sciences 366 (1881): 3717-25. https://doi.org/10.1098/ rsta.2008.0118. 